OnLine Journal of Biological Sciences 10 (2): 84-91, 2010

ISSN 1608-4217

C) 2010 Science Publications

\title{
Cognitive-Enhancing and Antioxidant Activities of Quercetin Liposomes in Animal Model of Alzheimer's Disease
}

\author{
${ }^{1}$ Terdthai Tong-un, ${ }^{1}$ Panakaporn Wannanon, ${ }^{1}$ Jintanaporn Wattanathorn and ${ }^{2}$ Wathita Phachonpai \\ ${ }^{1}$ Department of Physiology, Faculty of Medicine, Khon Kaen University, Khon Kaen, Thailand, 40002 \\ ${ }^{2}$ Department of Physiology, Faculty of Medicine, (Neuroscience Program) and Graduate School, \\ Khon Kaen University, Thailand, 40002
}

\begin{abstract}
Problem statement: Recent findings demonstrated the crucial role of oxidative stress on the pathophysiology of Alzheimer's Disease (AD). Antioxidant intake is beneficial to delay or inhibit the progression of this disease. Quercetin, a bioflavonoid in fruits and vegetables, has a powerful antioxidant activity both in vitro and in vivo. Claims that quercetin has many biological activities. However, quercetin is rapidly metabolized and limited ability to cross the blood-brain-barrier are obstacles to its use for treatment of AD. Liposomes have been used as an effective delivery system to the brain. Advantages associated with the nasal administration over oral route include higher bioavailability due to no first pass hepatic metabolism and rapid absorption leading to shorter time to onset of effect. Based on this information, the effects of quercetin liposomes via nasal route on improving cognitive behavior and biochemical markers of oxidative stress, Superoxide Dismutase (SOD), catalase, glutathione and Malondialdehyde (MDA) in the hippocampus in animal model of AD were investigated. Approach: Male Wistar rats were pretreated with quercetin liposomes, containing $0.5 \mathrm{mg}$ of quercetin in $20 \mu \mathrm{L}$ (dose $=20 \mu \mathrm{g}$ ), via intranasal route once daily continually for 2 weeks before and 1 week after AF64A administration. Learning and memory was evaluated using the Morris water maze test at 7 days after the AF64A administration and then the rats were sacrificed for determining the content of MDA and the activities of SOD, catalase and glutathione in the hippocampus. Results: Quercetin liposomes via nasal administration significantly improved memory impairment by inhibiting the oxidative damage in hippocampus. The possible underlying mechanisms might be partly associated with the decrease the level of MDA whereas increase the activity of SOD, catalase and glutathione. Conclusion: Our studies demonstrated that quercetin liposomes via nasal administration may have a therapeutic importance in the clinical management of AD.
\end{abstract}

Key words: Quercetin liposomes, nasal administration, spatial memory, oxidative stress

\section{INTRODUCTION}

Reactive Oxygen Species (ROS), such as superoxide anion, hydroxyl radical and hydrogen peroxide, have a causal relationship with oxidative stress. Many studies have demonstrated that overproduction of ROS can further aggravate the oxidative stress and the result is the pathogenesis of Alzheimer's Disease (AD) (Markesbery, 1999; Bokov et al., 2004). AD is a progressive and complex neurodegenerative disease, characterized by progressive decline in memory, language and other cognitive functions (Auld et al., 2002). It has been observed that the use of antioxidants as well as dietary improvements with regard to the consumption of fruits and vegetables high in antioxidant activity and neuroprotective agents may decrease the risk of memory deficits of the AD type (Weinstock and Shoham, 2004).

Quercetin (3,5,7,3',4'-pentahydroxyflavone), a bioflavonoid, frequently found in consumed foods including apples, berries, onions, tea and vegetables (Formica and Regelson, 1995). Indeed, quercetin has many beneficial effects on human health, including cardiovascular protection, anticancer activity, cataract prevention, antiviral activity and antiinflammatory effects (Nagata et al., 1999). Despite extensive research on the beneficial effects of quercetin in various pathological conditions, the idea of applying quercetin as the agent to improve memory deficit in Alzheimer's condition has become attractive. In order to apply quercetin for this case, it is necessary to overcome the limitations of quercetin: Poor absorption and very low

Corresponding Author: Terdthai Tong-un, Department of Physiology, Faculty of Medicine, Khon Kaen University, Khon Kaen 40002, Thailand Tel: 66-43-348394 
distribution to the brain after oral administration (Boer et al., 2005) due to both rapid metabolism (Manach et al., 1998) and difficulties in the penetration through the Blood-Brain Barrier (BBB) (Youdim et al., 2004).

Liposomes have long been used as a Drug Delivery System (DDS) to the brain, because the particles entrap the compounds and prevent rapid degradation elimination or elimination as well as promote penetration through the $\mathrm{BBB}$ and distribution in the brain tissue (Krauze, 2006). Being entrapped in liposomes, a decrease in the dose of a compound to be administered is usually expected (Keller, 2001).

Nasal administration has been proposed as a noninvasive method to deliver bioactive substances to the brain. This route of administration has the potential of decreased hepatic metabolism and greater access to the brain tissue through the olfactory nerve compared to the oral route (Wang et al., 2006) and could be a potential method of delivering quercetin to the Central Nervous System (CNS). Therefore, this provides more opportunity for quercetin to enter the CNS and then act on CNS to improve learning and memory with highly efficiency. In view of this, the present study was undertaken to investigate the effects of quercetin liposomes via nasal route on cognitive dysfunction and associated oxidative damage in animal model of AD.

\section{MATERIALS AND METHODS}

High-purity egg L- $\alpha$-phosphatidylcholine, type XVI-E (EPC), cholesterol (chol), quercetin dihydrate (98\% HPLC purity) and Polyethylene Glycol 400 (PEG) were purchased from Sigma (Barcelona, Spain). Other reagents used were analytical grade such as chloroform, ethanol and methanol (HPLC and analytical grade) from BDH Laboratory Supplies (Poole, England), disodium hydrogen phosphate, perchloric acid and ortho phosphoric acids (Merck, Darmstadt, Germany), sodium dihydrogen phosphate (JT Baker Inc., Phillipsburg, New Jersey). All other chemicals were at least reagent grade and used as received.

Preparation of quercetin liposomes: Quercetin dehydrate (98\%), high-purity egg L- $\alpha$ phosphatidylcholine, Type XVI-E (EPC) and Cholesterol (CHOL) were prepared as quercetin liposomes. The method used was lipid thin film formation and extrusion (Guo et al., 2003; Liang et al., 2005; Zu et al., 2006).

Animals: Adult male Wistar rats $(180 \pm 20 \mathrm{~g}, 8$ weeks old) were obtained from National Animal Center,
Salaya, Nakhon Pathom and they were housed in group of five per cage in standard metal cages at $22 \pm 2^{\circ} \mathrm{C}$ on 12:12 h light-dark cycle. All animals were given access to food and water ad libitum. The experiments were performed to minimize animal suffering in accordance with the internationally accepted principles for laboratory use and care of European Economic Community (EEC directive of 1986; 86/609/EEC).

The experimental protocols were approved by the Institutional Animal Care and Use Committee.

Experimental protocol: All rats were randomly assigned to five groups of eight animals each:

- Free liposomes + ACSF: the rats in this group were administered free liposomes via nasal route then they were administered artificial cerebrospinal fluid or ACSF bilaterally via intracerebroventricular route

- PEG + ACSF: the rats in this group were administered PEG which used as vehicle of quercetin liposomes via nasal route and subjected to ACSF administration

- Free liposomes + AF64A: all rats were administered free liposomes via nasal route then, they were administered AF64A bilaterally via intracerebroventricular route

- PEG + AF64A: the animals were administered PEG via nasal route then they were administered AF64A as mentioned in group 3

- $\mathrm{QCL}+\mathrm{AF} 64 \mathrm{~A}$ : the rats in this group were administered quercetin liposomes via nasal route, then they were administered AF64A

All animals were administered freshly prepared quercetin liposomes, containing $0.5 \mathrm{mg}$ of quercetin in $20 \mu \mathrm{L}($ dose $=20 \mu \mathrm{g})$, or liposomes without quercetin at the same volume via right nasal cavity with a micropipette at a period of 2 weeks before and 1 week after AF64A or Artificial Cerebrospinal Fluid (ACSF) administration. The animals were determined the spatial memory 1 week after AF64A administration then they were sacrificed for determined the activity of SOD, catalase, glutathione and the level of MDA in the hippocampus.

AF64A administration: AF64A was prepared as described previously by Hanin (1996). Briefly, an aqueous solution of acetylethylcholine mustard $\mathrm{HCl}$ (Sigma, St. Louis, MO) was adjusted to $\mathrm{pH} 11.3$ with $\mathrm{NaOH}$. After stirring for $30 \mathrm{~min}$ at room temperature, the $\mathrm{pH}$ was lowered to 7.4 with the gradual addition of $\mathrm{HCl}$ and stirred for $60 \mathrm{~min}$. The amount of AF64A was 
then adjusted either to $2 \mathrm{nmol} / 2 \mu \mathrm{L}$. The vehicle of AF64A was distilled water prepared in the same manner as the AF64A and recognized as ACSF. In order to administer AF64A bilaterally via intracerebroventricular (i.c.v.) route, the animals were anesthetized with the intraperitoneal injection of sodium pentobarbital at dose of $60 \mathrm{mg} \mathrm{kg}^{-1} \mathrm{BW}$. Then, AF64A ( $2 \mathrm{nmol} / 2 \mu \mathrm{L})$ was infused bilaterally via intracerebroventricular (i.c.v.) route with a 30-gauge needle inserted through a burr hole drilled into the skull into both the right and left lateral ventricles. Stereotaxic coordinates were (from the bregma): Posterior $0.8 \mathrm{~mm}$, lateral $\pm 1.5 \mathrm{~mm}$ and ventral (from dura) $3.6 \mathrm{~mm}$. The rate of infusion was $1.0 \mu \mathrm{L} \mathrm{min}{ }^{-1}$ and the needle was left in place for $5 \mathrm{~min}$ after infusion and then slowly withdrawn.

\section{Behaviors evaluation:}

Morris water maze test: The water maze consisted of a metal pool $(170 \mathrm{~cm}$ in diameter $\times 58 \mathrm{~cm}$ tall $)$ filled with tap water $\left(25^{\circ} \mathrm{C}, 40 \mathrm{~cm}\right.$ deep) divided into four quadrants. In the center of one quadrant was a removable escape platform below the water level and covered with a nontoxic milk powder. The pool was divided into four quadrants (NE, NW, SE and SW) by two imaginary lines crossing the center of the pool. For each animal, the location of invisible platform was placed at the center of one quadrant and remained there throughout training. The rats must memorize the platform location in relation to various environmental cues because there was nothing directly shows the location of the escape platform in and outside the pool. Therefore, the placement of the water tank and platform were the same in all acquisition trials. Each rat was gently placed in the water facing the wall of the pool from one of the four starting points (N, E, S, or W) along the perimeter of the pool and the animal was allowed to swim until it found and climbed onto the platform. During training session, the subject was gently placed on the platform by the experimenter when it could not reach the platform in $60 \mathrm{sec}$. In either case, the subject was left on the platform for $15 \mathrm{sec}$ and removed from the pool. The time for animals to climb on the hidden platform was recorded as escape latency or acquisition time. In addition to the acquisition test, the determination of retention memory was carried out on the next day. According to this test, the platform was removed and the animals were placed into the water maze for $60 \mathrm{sec}$. The retention of memory or the time that the animal spent to swim around the previous location of platform before removing the platform on the test occurring in the next day was also recorded. It has been postulated that if the spatial memory of the rat for the trained platform location is accurate, the rat will swim to the platform location and search around the exact location. Therefore, the more accurate the spatial memory is, the greater the number of times rat swim across the trained platform. In each trial, the animal was quickly dried with towel before being returned to the cage. All test in Morris water maze tests were carried out within 30 min after the nasal administration of the substances.

Any enhancement of cognition would be reflected by a decrease in escape latency and increase in retention time.

\section{Estimation of oxidative stress parameters:}

Tissue preparation: Brain tissue samples were thawed and homogenized with 10 times (w/v) by homogenizer in ice-cold 0.1 M phosphate buffer ( $\mathrm{pH} 7.4$ ). Aliquots of homogenates from rat brain were separated and used to determine protein, MDA, SOD, catalase and glutathione. Whereas the remaining homogenates were centrifuged at 15,000 rpm for $60 \mathrm{~min}$ and supernatant was then used for enzyme assays. Catalase activity was determined immediately after sample preparation and SOD was determined within $24 \mathrm{~h}$. Protein concentration was determined according to Lowry et al. (1951) using purified bovine serum albumin as standard.

Measurement of lipid peroxidation: MDA, which is a measure of lipid peroxidation, was measured spectrophotometrically (Ohkawa et al., 1979). Briefly, brain tissues were homogenized with 10 times (w/v) $0.1 \mathrm{M}$ sodium phosphate buffer $(\mathrm{pH} 7.4)$. The reagents acetic acid $1.5 \mathrm{~mL}(20 \%) \mathrm{pH} 3.5,1.5 \mathrm{~mL}$ thiobarbituric acid $(0.8 \%)$ and $0.2 \mathrm{~mL}$ sodium dodecyl sulfate $(8.1 \%)$ were added to $0.1 \mathrm{~mL}$ of processed tissue sample. The mixture was then heated at $100^{\circ} \mathrm{C}$ for $60 \mathrm{~min}$. The mixture was cooled with tap water and $5 \mathrm{~mL}$ of $\mathrm{n}$ butanol: Pyridine (15:1\% v/v), $1 \mathrm{~mL}$ of distilled water was added. The mixture was shaken vigorously. After centrifugation at $4000 \mathrm{rpm}$ for $10 \mathrm{~min}$, the organic layer was withdrawn and absorbance was measured at $532 \mathrm{~nm}$ using a spectrophotometer.

Measurement of glutathione: Glutathione was measured spectrophotometrically (Ellman, 1959). Briefly, brain tissues were homogenized with 10 times (w/v) 0.1 M sodium phosphate buffer ( $\mathrm{pH} 7.4)$. This homogenate was then centrifuged with $5 \%$ trichloroacetic acid to centrifuge out the proteins. To $0.1 \mathrm{~mL}$ of this homogenate, $2 \mathrm{~mL}$ of phosphate buffer (pH 8.4), $0.5 \mathrm{~mL}$ of 505-Dithiobis (2-Nitrobenzoic acid) (DTNB) and $0.4 \mathrm{~mL}$ of double distilled water was added. The mixture was vortexed and the absorbance read at $412 \mathrm{~nm}$ within $15 \mathrm{~min}$. 
Measurement of catalase: Catalase activity was measured by the method of Goldblith and Proctor (1950). About $0.1 \mathrm{~mL}$ of supernatant was added to cuvette containing $1.9 \mathrm{~mL}$ of $50 \mathrm{mM}$ phosphate buffer (pH 7.0). Reaction was started by the addition of $1.0 \mathrm{~mL}$ of freshly prepared $30 \mathrm{mM} \mathrm{H}_{2} \mathrm{O}_{2}$. The rate of decomposition of $\mathrm{H}_{2} \mathrm{O}_{2}$ was measured spectrophotometrically from changes in absorbance at $240 \mathrm{~nm}$.

Measurement of SOD: SOD activity was measured according to the method described by McCord and Fridovich (1969). Solution A was prepared by mixing $100 \mathrm{~mL}$ of $50 \mathrm{mM}$ PBS (pH 7.4) containing $0.1 \mathrm{mM}$ EDTA and $2 \mu \mathrm{mol}$ of cytochrome $\mathrm{c}$ with $10 \mathrm{~mL}$ of $0.001 \mathrm{~N} \mathrm{NaOH}$ solution containing $5 \mu \mathrm{mol}$ of xantine. Solution B included $0.2 \mathrm{U}$ xantine oxidize $\mathrm{mL}^{-1}$ and $0.1 \mathrm{~mm}$ EDTA $50 \mu \mathrm{L}$ of a tissue supernatant were mixed with $2.9 \mathrm{~mL}$ of solution $\mathrm{A}$ and the reaction was started by adding $50 \mu \mathrm{L}$ of solution B. change in absorbance at $550 \mathrm{~nm}$ was monitored.

Statistical analysis: Data are presented as mean \pm standard error of mean $( \pm$ SEM). One-way Analysis Of Variance (ANOVA), followed by Duncan's post hoc test. A probability level less than 0.05 was accepted as significance.

\section{RESULTS}

Effect of quercetin liposomes on spatial memory in AF64A treated rats: Figure 1 and 2 shows that ACSF produced no significant changes on both escape latency and retention time in Morris water maze test. Intracerebroventricular administration of AF64A significantly increased escape latency but decreased retention time ( $\mathrm{p}$-value $<0.001$ all; compared to both liposomes + ACSF and PEG + ACSF). This indicated the memory impairment induced by AF64A. Rats that received AF64A and nasal administration of quercetin liposomes showed significantly decreased acquisition and increased retention time ( $p$-value $<0.001$ all; compared to liposomes + ACSF and PEG + AF64A). Therefore, our data indicated that quercetin liposomes via nasal administration improved the memory deficit induced by AF64A.

Effect of quercetin liposomes on MDA level, SOD, catalase and glutathione activities in AF64A treated rats: In order to determine the possible underlying mechanism of quercetin liposomes, we had determined the effect of quercetin liposomes on oxidative stress indices including the level of Malondialdehide (MDA), a product of lipid peroxidation product and the activities of scavenger enzymes including SOD, catalase and glutathione in hippocampus as shown in Table 1. Intracerebroventricular administration of ACSF had no effect on brain MDA levels or SOD catalase and glutathione, whereas AF64A administration caused a marked increase in free radical generation and a significant rise in brain MDA levels and depletion of SOD, catalase and glutathione (p-value<0.001 all; compared to both liposomes + ACSF and PEG + ACSF).

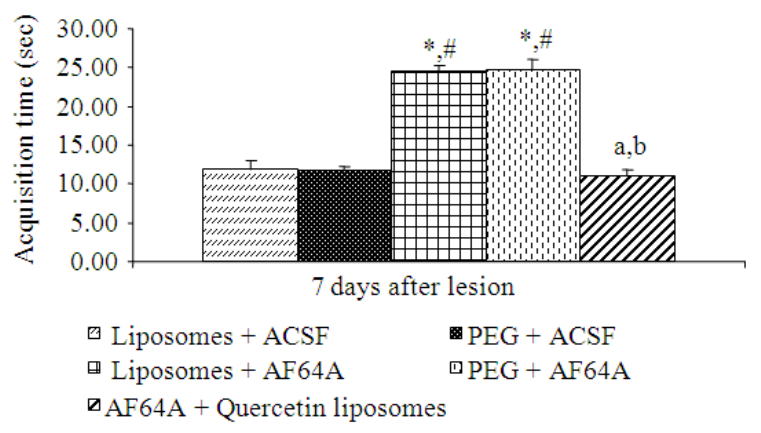

Fig. 1: Effect nasal administration of quercetin liposomes on the acquisition time of spatial learning in the Morris water maze test $(\mathrm{N}=8)$. Results were expressed as mean \pm SEM. *: p-value $<0.001$ compared with liposomes + ACSF treated group, \#: p-value $<0.001$ compared with PEG + ACSF treated group, a: $p$-value $<0.001$ compared with liposomes + AF64A treated group, b: p-value $<0.001$ compared with PEG + AF64A treated group

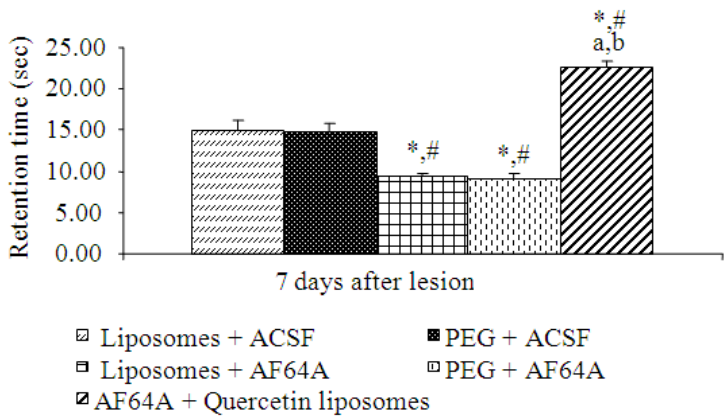

Fig. 2: Effect nasal administration of quercetin liposomes on the retention time of spatial learning in the Morris water maze test $(\mathrm{N}=8)$. Results are expressed as mean \pm SEM. *: p-value $<0.001$ compared with liposomes + ACSF treated group, ": p-value $<0.001$ compared with $\mathrm{PEG}+\mathrm{ACSF}$ treated group, a: p-value $<0.001$ compared with liposomes + AF64A treated group, ${ }^{b}$ : p-value $<0.001$ compared with PEG + AF64A treated group 
OnLine J. Biol. Sci., 10 (2): 84-91, 2010

Table 1: Effect nasal administration of quercetin liposomes on the activities of SOD, catalase, glutathione and the levels of lipid peroxidation in animal model of AD induced by AF64A

\begin{tabular}{lllll}
\hline Experimental conditions & $\mathrm{SOD}\left(\mathrm{U} \mathrm{mg}^{-1}\right.$ protein $)$ & Catalase $\left(\mathrm{U} \mathrm{mg}^{-1}\right.$ protein $)$ & Glutathione $\left(\mathrm{U} \mathrm{mg}^{-1} \mathrm{protein}\right)$ & $\mathrm{MDA}\left(\mathrm{U} \mathrm{mg}^{-1}\right.$ protein $)$ \\
\hline Liposomes + ACSF & $16.80 \pm 0.13$ & $18.10 \pm 4.55$ & $14.44 \pm 0.12$ & $1.45 \pm 0.11$ \\
PEG + ACSF & $16.41 \pm 1.27$ & $18.67 \pm 3.84$ & $14.41 \pm 0.11$ & $1.48 \pm 0.17$ \\
Liposomes + AF64A & $11.20 \pm 0.17^{* \#}$ & $16.14 \pm 1.48^{* \#}$ & $11.20 \pm 0.47^{* \#}$ & $2.48 \pm 0.27^{* \#}$ \\
PEG + AF64A & $12.24 \pm 1.90^{* \#}$ & $16.00 \pm 1.73^{* \#}$ & $11.87 \pm 0.22^{* \#}$ & $2.49 \pm 0.26^{* \#}$ \\
QCL + AF64A & $22.50 \pm 0.12^{* \#, \mathrm{a}, \mathrm{b}}$ & $19.80 \pm 3.43^{* \#, \mathrm{a}, \mathrm{b}}$ & $18.31 \pm 0.33^{* \#, \mathrm{a}, \mathrm{b}}$ & $1.19 \pm 0.13^{* \#, \mathrm{a}, \mathrm{b}}$ \\
\hline
\end{tabular}

Results are expressed as mean \pm SEM; ; : p-value $<0.001$ compared with liposomes + ACSF treated group; ${ }^{\#}:$ p-value $<0.001$ compared with PEG+ACSF treated group; ${ }^{\mathrm{a}}$ : p-value $<0.001$ compared with liposomes + AF64A treated group; ${ }^{\text {b: }}$ p-value $<0.001$ compared with PEG + AF64A treated group

Surprisingly, treatment with quercetin liposomes exerted complete protection to these enzymes and decreased the lipid peroxidation in rat brain changes $(\mathrm{p}-$ value $<0.001$ all; compared to liposomes + AF64A and PEG + AF64A).

\section{DISCUSSION}

The present study examined the effect of quercetin liposomes via nasal administration on memory impairment and oxidative stress in animal model of Alzheimer's disease induced by AF64A.

Recently, numerous lines of evidence have demonstrated that oxidative stress is associated with the pathogenesis of $\mathrm{AD}$ and cellular characteristics of this disease are either causes or effects of oxidative stress (Mattson, 2004). During the last few years, antioxidant has received special attention as dietary supplements. Many studies have suggested that reversals in agerelated memory declines might be accomplished by increasing the dietary intake possessing high antioxidant activity (Galli et al., 2002; Andres-Lacueva et al., 2005; Jabeen et al., 2007) and this reversal effect has been claimed to be associated with the antioxidant activity (Raghavendra and Kulkarni, 2001). Thus, many researchers focused on the beneficial effects of supplement possessing a capability to improve antioxidant activity were considered to be a potential candidate for neuroprotective agent against AD.

Quercetin, a main flavonoid found in fruits, vegetables and beverages, was reported to possess antioxidant and cognition (Boots et al., 2008; Reiter et al., 2009). Previous studies confirm that quercetin supplementation improve memory deficit condition induced by reserpine in mice (Naumenko and Kulikov, 2006). However, it was reported that quercetin was easily metabolized after absorption (Manach et al., 2004). Our study found it worthwhile to use the vesicle mediated carrier system particularly liposomes to encapsulated the quercetin against Alzheimer's condition induced by AF64A as well as oxidative stress.
Intracerebroventricular injection by AF64A has been described as an appropriate the substance to induce memory deficit via increase the oxidative stress in all area of hippocampus, the areas contribution important role on learning leading to the neurodegeneration in the mentioned areas resulting in learning and memory impairment as those observed in AD (Chrobak et al., 1988). Based on the correspondence changes of behavior and neuropathology induced by AF64A and AD, this study used AF64A to develop animal model of AD.

In the present study, the results show that both in the AF64A + free liposomes and AF64A + PEG treated groups, caused a persistent memory deficit as evidenced by a significant induction in acquisition time and reduction in retention time in Morris water maze. Moreover, our results show that in the AF64A with nasal administration of free liposomes and AF64A with nasal administration of PEG treated groups, there were the reduction of scavenging enzymes activities including SOD, catalase and glutathione, while enhanced the level of MDA, which is an indicative of lipid peroxidation were appear in these groups. These results are in conformity with other workers, who have demonstrated cognitive impairment after induced by AF64A in rats (McDonald and Overmier, 1998; Gulyaeva et al., 1996).

No unexpected mortality of any animals occurred after nasal administration of quercetin liposomes used in the present study. As a result, vesicles were considered to be safe at the dosing schedule used. Interestingly, the rats was treated with quercetin liposomes via nasal route showed significantly decreased acquisition and increased retention time in Morris water maze test as compared to the liposomes + AF64A and PEG + AF64A treated animals, suggested its cognitive enhancing effect. Moreover, pretreatment with nasal administration of quercetin liposomes significantly reversed the impact of oxidative alterations (MDA, SOD, catalae and glutathione) seen in Alzheimer's condition induced by AF64A; this shows the antioxidant potential of quercetin liposomes via nasal administration. 
The mechanism how quercetin liposomes could decrease lipid peroxidation and improve its antioxidant systems against Alzheimer's condition induced by AF64A is not known, but it may be presumed that after entering the brain, the quercetin or its derivative might increase the activities of scavenger enzymes mentioned earlier and resulted in the decrease excess free radicals, which in turn decreased the lipid peroxidation process leading to the reduction of MDA as shown in this study. These results are in accordance with the report of quercetin liposomes effect to decrease lipid peroxidation and increase scavenger enzymes activities in rat brain by the induction of cerebral ischemia and reperfusion (Sarkar and Das, 2006). However, the method to prepare the quercetin liposomes and the model to induce the oxidative damage were different.

Our results demonstrated that nasal administration of quercetin encapsulated liposomes was a potential novel strategy to protect against neurodegeneration disorder such as AD. It was noticeable that the dose of quercetin required via nasal administration was very much low dose and rapid onset of cognitive enhancing effect. In this respect, quercetin could be delivered to the brain even though it was administered in nondissolved form in a liquid that was not a good candidate for delivery of this hydrophobic compound (Cho et al., 2006). Some of the suspended particles of quercetin might not be absorbed after all. Our present study suggests that the enhanced delivery of quercetin in the form of liposomes to the brain could effectively reduce the dose, which would also reduce the potential of toxicity of the substance (Metodiewa et al., 1999) and increase bioavailability (Jamal et al., 2000). Moreover, it was also provided numerous benefits especially the direct nose-to-brain delivery, bypassing the blood brain barrier (Wu et al., 2008) and avoid the first pass metabolism (Graf et al., 2006). However, the main proposed pathway of quercetin delivery via nasal administration was different. It was likely to permeate through the subarachnoid space through the olfactory epithelium and found in the CSF later, because the liposomes behaved as semilipophilic particles. Therefore, quercetin liposomes could rapidly absorb into the CSF. Investigation of quercetin absorption and distribution after administered via nasal route would be of value for future studies.

\section{CONCLUSION}

Nasal administration of quercetin liposomes prevents AF64A-induced cognitive impairment and associated with oxidative stress. Further, the use of quercetin liposomes via nasal route is warrants evaluation for the treatment of neurological disorders, which are associated with free radical generation and cognitive impairment such as AD.

\section{ACKNOWLEDGEMENT}

This study was partial supported by the National Nanotechnology Center (NANOTEC), NSTDA, National Science and Technology Development Agency, Thailand, through its program of Center of Excellence Network.

\section{REFERENCES}

Andres-Lacueva, C., B. Shukitt-Hale, R.L. Galli O. Jauregui and R.M. Lamuela-Raventos et al., 2005. Anthocyanins in aged blueberry-fed rats are found centrally and may enhance memory. Nutr. Neurosci., 8: 111-120. PMID: 16053243

Auld, D.S., T.J. Kornecook, S. Bastianetto and R. Quirion, 2002. Alzheimer's disease and the basal forebrain cholinergic system: Relations to $\beta$-amyloid peptides cognition and treatment strategies. Prog. Neurobiol., 68: 209-245. PMID: 12450488

Boer, V.C.J., A.A. Dihal, H. van Derwoude, I.C.W. Arts and S. Wolffram et al., 2005. Tissue distribution of quercetin in rats and pigs. J. Nutr., 135: 1617-1618. PMID: 15987855

Bokov, A., A. Chaudhuri and A. Richardson, 2004. The role of oxidative damage and stress in aging. Mech. Ageing Dev., 125: 811-826. PMID: 15541775

Boots, A.W., G.R. Haenen and A. Bast, 2008. Health effects of quercetin: From antioxidant to nutraceutical. Eur. J. Pharmacol., 585: 325-337. DOI: 10.1016/j.ejphar.2008.03.008

Cho, J.Y., I.S. Kim, Y.H. Jang, A.R. Kim and S.R. Lee, 2006. Protective effect of quercetin, a natural flavonoid against neuronal damage after transient global cerebral ischemia. Neurosci. Lett., 404: 330-335. PMID: 16806698 DOI: 10.1016/j.neulet.2006.06.010

Chrobak, J.J., I. Hanin, D.E. Schmechel and T.J. Walsh, 1988. AF64Ainduced working memory impairment: Behavioral, neurochemical and histological correlates. Brain Res., 463: 107-117. DOI: 10.1016/0006-8993(88)90532-X

Ellman, G.L., 1959. Tissue sulfhydryl groups. Arch. Biochem. Biophys., 82: 70-77. PMID: 13650640

Formica, J.V. and W. Regelson, 1995. Review of the biology of quercetin and related bioflavonoids. Food Chem. Toxicol., 33: 1061-1080. PMID: 8847003 
Galli, R.L., B. Shukitt-Hale, K.A. Youdim and J.A. Joseph, 2002. Fruit polyphenolics and brain aging: Nutritional interventions targeting age-related neuronal and behavioral deficits. Ann. N. Y. Acad. Sci., 959: 128-132. PMID: 11976192

Goldblith, S.A. and B.E. Proctor, 1950. Photometric determination of catalase activity. J. Biol. Chem., 187: 705-709. PMID: 14803454

Graf, B.A., C. Ameho, G.G. Dolnikowski, P.E. Milbury and C.Y. Chen et al., 2006. Rat gastrointestinal tissues metabolized quercetin. J. Nutr., 136: 39-44. PMID: 16365056

Gulyaeva, N., N. Lazareva, M. Libe, O. Mitrokhina and M. Onufriev et al., 1996. Oxidative stress in the brain following intraventricular administration of ethylcholine aziridinium (AF64A). Brain Res., 726: 174-180. DOI: 10.1016/0006-8993(96)00330-7

Guo, J., Q. Ping, G. Jiang, L. Huang and Y. Tong, 2003. Chitosan-coated liposomes: Characterization and interaction with leuprolide. Int. J. Pharm., 260: 167-173. DOI: $10.1016 / \mathrm{S} 0378-$ 5173(03)00254-0

Hanin, I., 1996. The AF64A model of cholinergic hypofunction: An update. Life Sci., 58: 1955-1964. DOI: 10.1016/0024-3205(96)00185-3

Jabeen, B., M. Badaruddin, R. Ali and D.J. Haleem, 2007. Attenuation of restraint induced behavioral deficits and serotonergic responses by stabilized rice bran in rats. Nutr. Neurosci., 10: 11-16. PMID: 17539478

Jamal, T., S. Jean-Michel, R.R. Anthony and K. Michel, 2000. Brain drug delivery technologies: Novel approaches for transporting therapeutics. Pharm. Sci. Technol., 3: 155-162. PMID: 10785657

Keller, B.C., 2001. Liposomes in nutrition. Trends. Food Sci. Technol., 12: 25-31. DOI: 10.1016/S0924-2244(01)00044

Krauze, L., 2006. Real-time imaging and quantification of brain delivery of liposomes. Pharm. Res., 23: 2493 2504. DOI: 10.1007/s11095-006-9103-5

Liang, M.T., N.M. Davies and I. Toth, 2005. Encapsulation of lipopeptides within liposomes: Effect of number of lipid chains, chain length and method of liposome preparation. Int. J. Pharm., 301: 247-254. PMID: 16054787

Lowry, O.H., N.J. Rosenbrough, A.L. Farr and R.J. Randall, 1951. Protein measurement with folin phenol reagent. J. Biol. Chem., 193: 265-75. PMID: 14907713

Manach, C., C. Morand, V. Crespy, C. Demignee and O. Texier et al., 1998. Quercetin is recovered in human plasma as conjugated derivatives which retain antioxidant properties. FEBS Lett., 426: 331-336. PMID: 9600261
Manach, C., A. Scalbert, C. Morand, C. Remesy and L. Jimenez, 2004. Polyphenols: Food sources and bioavailability. Am. J. Clin. Nutr., 79: 727-747. PMID: 15113710

Markesbery, W.R., 1999. Oxidative stress hypothesis in Alzheimer's disease. Free Radic. Biol. Med., 23: 134-147. PMID: 9165306

Mattson, M.P., 2004. Pathways towards and away from Alzheimer's disease. Nature, 430: 631-639.

McCord, J.M. and I. Fridovich, 1969. Superoxide dismutase. An enzymic function for erythrocuprein (hemocuprein). J. Biol. Chem., 244: 6049-6055. PMID: 5389100 DOI: 10.1038/nature02621

McDonald, M.D. and J.B. Overmier, 1998. Present imperfect: A critical review of animal models of the mnemonic impairments in Alzheimer's disease. Neurosci. Biobehav. Rev., 22: 99-120. DOI: 10.1016/S0149-7634(97)00024-9

Metodiewa, D., A.K. Jaiswal, N. Cenas, E. Dickancaite and J. Segura-Aguilar, 1999. Quercetin may act as a cytotoxic prooxidant after its methanolic activation to semiquinone and quinoidal product. Free Radic. Biol. Med., 26:107-116. PMID: 9890646

Nagata, H., S. Takekoshi, T. Honna and K. Watanabe, 1999. Antioxidative action of flavonoids quercetin and catachin mediated by the activation of glutathione peroxidase. J. Exp. Clin. Med., 24: 1-11. PMID: 10530620

Naumenko, V.S. and A.V. Kulikov, 2006. Quantitative assay of 5-HT (1A) serotonin receptor gene expression in the brain. Molekuliarnaia Biol., 40: 37-44. PMID: 16523690

Ohkawa, H., N. Ohishi and K. Yagi, 1979. Assay for lipid peroxide in animal tissues by thiobarbituric acid reaction. Anal. Biochem., 95: 351-358. DOI: 10.1016/0003-2697(79)90738-3

Raghavendra, V. and S.K. Kulkarni, 2001. Possible antioxidant mechanism in melatonin reversal of aging and chronic ethanol-induced amnesia in plusmaze and passive avoidance memory tasks. Free Radic. Biol. Med., 30: 595-602. PMID: 11295357

Reiter, M., K. Rupp, P. Baumeister, S. Zieger and U. Harreus, 2009. Antioxidant effects of quercetin and coenzyme Q10 in mini organ cultures of human nasal mucosa cells. Anticancer Res., 29: 33-39. PMID: 19331131

Sarkar, S. and N. Das, 2006. Mannosylated liposomal flavonoid in combating age-related ischemiareperfusion induced oxidative damage in rat brain. Mech. Ageing Dev., 127: 391-397. DOI: 10.1016/j.mad.2005.12.010 
Wang, X., H. He, W. Leng and X. Tang, 2006. Evaluation of brain-targeting for the nasal delivery of estradiol by the microdialysis method. Int. J. Pharm., $\quad 317$ : 40-46. $\quad$ DOI: 10.1016/j.ijpharm.2006.02.055

Weinstock, M. and S. Shoham, 2004. Rat models of dementia based on reductions in regional glucose metabolism, cerebral blood flow and cytochrome oxidase activity. J. Neural Transm., 111: 347-366. PMID: 14991459

Wu, H., K. Hu and X. Jiang, 2008. From nose to brain: Understanding transport capacity and transport rate of drugs. Expert Opin. Drug Deliv., 5: 1159-1168. PMID: 18817519
Youdim, K.A., M.Z. Qaiser, D.J. Begley, C.A. Rice-Evans and N.J. Abbott, 2004. Flavonoid permeability across an in situ model of the blood-brain barrier. Free. Radic. Biol. Med., 36: 592-604. PMID: 14980703

Zu, Y., C. Li, Y. Fu and C. Zhao, 2006. Simultaneous determination of catechin, rutin, quercetin kaempferol and isorhamnetin in the extract of sea buckthorn (Hippophae rhamnoides L.) leaves by RP-HPLC with DAD. J. Pharm. Biomed. Anal., 41: 714-719. PMID: 16520013 DOI: 10.1016/j.jpba.2005.04.052 